\title{
Nearly a decade on - trends, risk factors and policy implications in global obesity
}

Vasanti S. Malik ${ }^{1,2}$, Walter C. Willet ${ }^{2,3,4,5}$ and Frank B. $H u^{2,3,4,5 凶}$

In the past decade, the prevalence of obesity and related comorbidities has continued to increase across the globe, prompting many countries to adopt policies to improve diet quality. Here, we discuss key nutrition policies that have been implemented in the past few years and consider future priorities for global obesity prevention.

In October 2011, Joana Osório, Chief Editor of Nature Reviews Endocrinology at the time, invited us to write a Review on the epidemiology of obesity and related comorbidities that would also discuss policy initiatives to tackle this immense public health challenge ${ }^{1}$. The paper was timely as obesity prevalence had more than doubled across the globe since 1980. High-income countries remained at the forefront of the epidemic, while similar trends had started to emerge in many low-income and middle-income countries (LMICs) ${ }^{2}$. The invitation to write the Review also came soon after the first United Nations General Assembly high-level summit on the prevention and control of noncommunicable diseases worldwide.

A key message from our paper was that global free trade and the economic growth and urbanization experienced by many LMICs were major drivers of obesity trends as they created obesogenic environments that promoted unhealthy dietary changes and reductions in physical activity. Specific dietary changes were characterized by increases in the consumption of animal products, refined grains and added sugar, largely from sugar-sweetened beverages (SSBs), due to the increased availability of low-cost processed products that are low in nutritional quality and high in calories. Given the complex aetiology of obesity, another key message was the need for multiple coordinated and sustained strategies to improve diet and physical activity across different levels of governance to curtail rising obesity trends. We also acknowledged the policy challenges some countries might face with concurrent undernutrition and obesity.

We worked with the editorial team to ensure a global perspective that included obesity trends from around the world and discussion of policies implemented in different countries. The Assistant Editor also requested more of an economic perspective. This suggestion prompted us to discuss the declining cost of food as a contributor to the obesity epidemic. We also considered Kenneth Rogoff's idea that obesity is an inevitable consequence of an unregulated capitalistic food system in which companies compete to sell their products. Our Review was published a year after inception and we received numerous article requests at the time of publication. To date, the paper remains a staple on the reading lists of multiple courses at Harvard University, MA, USA. It was also a basis for the obesity chapter in the Disease Control Priorities third edition (DCP3) ${ }^{3}$.

Since the time of publication, the prevalence of obesity has continued to rise across the world. In our discussion of temporal trends, we showed increases in obesity prevalence between 1980 and 2008 in various world regions, which were most marked among women and in Latin America, North America, North Africa and the Middle East. In 2008, an estimated 500 million adults, or 10-14\% of the global population, had obesity ${ }^{2}$. By 2016, this estimate had increased to 671 million ${ }^{4}$. As shown in our updated figure, with slightly different region classifications to those that were used in our Review, obesity prevalence increased in all regions from 2008 to 2016 with the highest rates observed among men in high-income Western countries and among women in Central Asia, the Middle East and North Africa (FIG. 1). According to a study that reported national survey data from 2000 to 2018 in the USA, obesity prevalence has increased to over $42 \%$ among adults and the prevalence of severe obesity $\left(\mathrm{BMI} \geq 40 \mathrm{~kg} / \mathrm{m}^{2}\right)$ doubled to $9.2 \%$ over the study period ${ }^{5}$. Published in 2019 , a study by the NCD risk Factor Collaboration found that BMI has risen at the same rate or faster in rural areas compared with cities in many LMICs, challenging the paradigm of urbanization as a key driver of the global obesity epidemic ${ }^{6}$. This finding has broad implications for nutrition policies that target undernutrition and obesity.

Over the past decade, in response to growing burdens of obesity and related chronic diseases, many countries have adopted policies that aim to improve diet quality with an emphasis on limiting the intake of SSBs, which are the largest source of added sugar in many populations. The World Health Organization guidelines (WHO guidelines) and the 2015 Dietary Guidelines for Americans (DGA) recommend limiting added sugar intake to no 


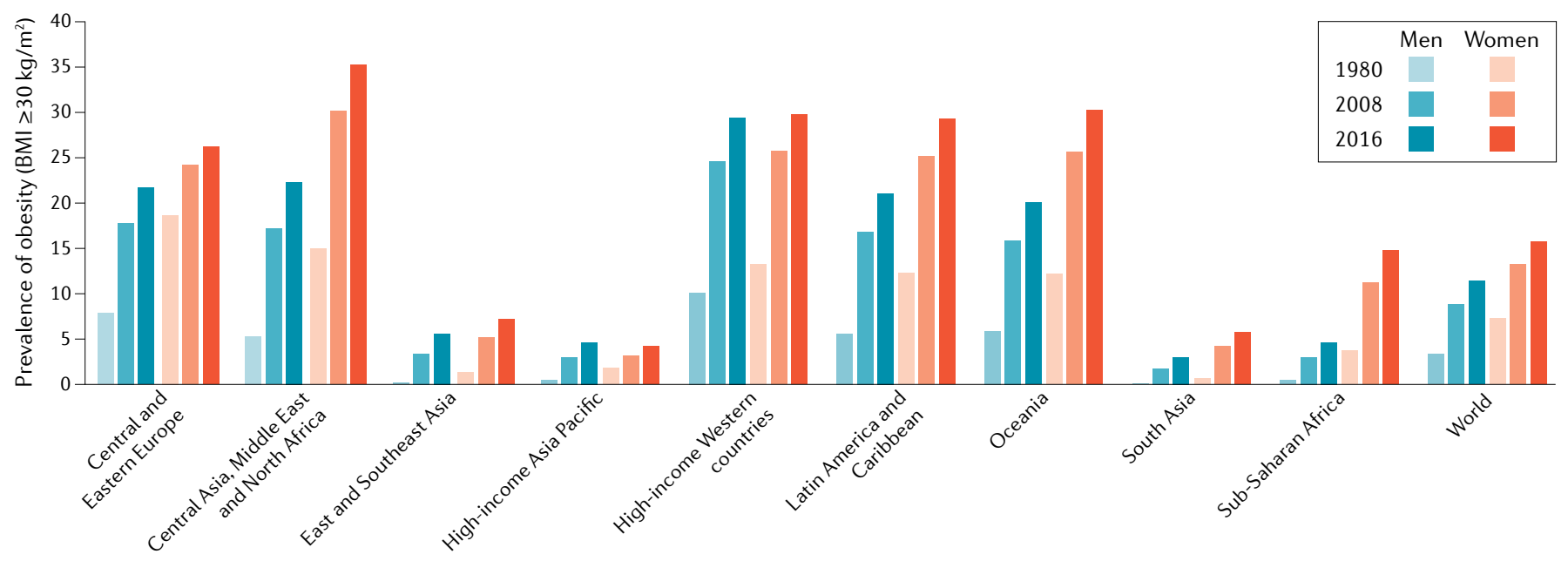

Fig. 1 | Global prevalence of obesity. Data on global trends in the prevalence of obesity $\left(B M I \geq 30 \mathrm{~kg} / \mathrm{m}^{2}\right)$ in men and women in 1980, 2008 and 2016 from select regions of the world show an increase in obesity across the world. Data from the Noncommunicable Diseases Risk Factor Collaboration (NCD-RisC).

more than $10 \%$ of caloric intake. Since our Review was published, several countries/regions and cities around the world have implemented taxes on SSBs as a strategy to reduce intake and generate revenue to support public health efforts. For instance, studies from Mexico and the USA have shown reductions in sales of SSBs and increases in sales of untaxed beverages such as water ${ }^{7,8}$. In addition, taxes on sugar led to reformulated beverages with reduced sugar content in the $\mathrm{UK}^{9}$. Whether these early benefits of SSB taxes will continue and translate into health improvements will be important to monitor.

In addition, several countries/regions have also adopted front-of-package nutrition labels to help consumers make healthy choices. Chile was the first to implement a warning label on foods that contain calories, sugar, saturated fat or sodium above certain thresholds. The introduction of similar warning labels is underway in Canada, Brazil, Uruguay and Israel. The revised nutrition facts panel in the USA now includes a line for added sugar with a corresponding percent daily value to help consumers meet dietary targets. Another major policy that has been implemented since the publication of our Review was the removal from the US food supply of artificial trans fats. According to the WHO, trans fats are responsible for over 500,000 premature deaths from cardiovascular disease each year worldwide. To address this issue, the WHO recently launched a campaign to replace trans fat in the global food supply by 2023 (WHO campaign).

Looking to the future, with the 2019 publication of the EAT-Lancet commission there is a growing global interest in diets that are primarily plant based, to benefit human and planetary health ${ }^{10}$. This trend is already reflected in industry innovation, with the proliferation of plant-based meat substitutes and alternative protein sources, such as insects, in the market. New frameworks are needed to evaluate the long-term health effects of consuming these products in place of more conventional foods. The importance of these obesity prevention measures has been greatly magnified by the current COVID-19 pandemic, as obesity and related chronic diseases are among the strongest predictors of COVID-19 severity and mortality.
Although we have seen some important steps aimed at reversing the global trends in obesity prevalence, the unrelenting increases indicate that our efforts have been insufficient. In the coming years, as the world grapples with rising obesity and chronic disease burdens alongside infectious disease epidemics, there will be an urgent need for coordinated actions across all countries/regions and sectors of society to prioritize the reduction of risk factors for obesity to ensure the health and welfare of the global population.

1. Malik, V. S., Willett, W. C. \& Hu, F. B. Global obesity: trends, risk factors and policy implications. Nat. Rev. Endocrinol. 9, 13-27 (2013).

2. Finucane, M. M. et al. National, regional, and global trends in body mass index since 1980: systematic analysis of health examination surveys and epidemiological studies with 960 country-years and 9.1 million participants. Lancet 377, 557-567 (2011).

3. Malik, V. S. \& Hu, F. B. in Disease Control Priorities 3rd edn Vol. 5 (eds Prabhakaran, D. et al.) (International Bank for Reconstruction and Development/The World Bank, 2017).

4. NCD Risk Factor Collaboration (NCD-RisC). Worldwide trends in body-mass index, underweight, overweight, and obesity from 1975 to 2016: a pooled analysis of 2416 population-based measurement studies in 128.9 million children, adolescents, and adults. Lancet 390, 2627-2642 (2017).

5. Hales, C. M., Carroll, M. D., Fryar, C. D. \& Ogden, C. L. Prevalence of obesity and severe obesity among adults: United States, 2017-2018. NCHS Data Brief 360, 1-8 (2020).

6. NCD Risk Factor Collaboration (NCD-RisC). Rising rural body-mass index is the main driver of the global obesity epidemic in adults. Nature 569, 260-264 (2019).

7. Colchero, M. A., Rivera-Dommarco, J., Popkin, B. M. \& Ng, S. W. In Mexico, evidence of sustained consumer response two years after implementing a sugar-sweetened beverage tax. Health Aff. 36, 564-571 (2017)

8. Silver, L. D. et al. Changes in prices, sales, consumer spending, and beverage consumption one year after a tax on sugar-sweetened beverages in Berkeley, California, US: a before-and-after study. PLoS Med. 14, e1002283 (2017).

9. Bandy, L. K., Scarborough, P., Harrington, R. A., Rayner, M. \& Jebb S. A. Reductions in sugar sales from soft drinks in the UK from 2015 to 2018. BMC Med. 18, 20 (2020).

10. Willett, W. et al. Food in the Anthropocene: the EAT-Lancet Commission on healthy diets from sustainable food systems. Lancet 393, 447-492 (2019).

Competing interests

The authors declare no competing interests.

\section{RELATED LINKS}

2015 Dietary Guidelines for Americans: https://health.gov/our-work/ food-nutrition/2015-2020-dietary-guidelines/guidelines/

Noncommunicable Diseases Risk Factor Collaboration (NCD-RisC) http://ncdrisc.org/data-downloads.html WHO campaign: https://www.who.int/nutrition/topics/replace-transfat WHO guidelines: https://www.who.int/publications/i/item/9789241549028 